\title{
Trivium
}

Revue franco-allemande de sciences humaines et sociales - Deutsch-französische Zeitschrift für Geistesund Sozialwissenschaften

$21 \mid 2016$

Autorégulation régulée. Analyses historiques de structures de régulation hybrides

\section{Regulierte Selbstregulierung. Historische Analysen hybrider Regelungsstrukturen \\ Einleitung}

Peter Collin et Sabine Rudischhauser

\section{CpenEdition}

Journals

Édition électronique

URL : http://journals.openedition.org/trivium/5245

ISSN : 1963-1820

Éditeur

Les éditions de la Maison des sciences de l'Homme

\section{Référence électronique}

Peter Collin und Sabine Rudischhauser, « Regulierte Selbstregulierung. Historische Analysen hybrider Regelungsstrukturen », Trivium [Online], 21 | 2016, online erschienen am 10 Mai 2016, abgerufen am 08 September 2020. URL : http://journals.openedition.org/trivium/5245

Ce document a été généré automatiquement le 8 septembre 2020.

\section{(c) $(1) \&$}

Les contenus des la revue Trivium sont mis à disposition selon les termes de la Licence Creative Commons Attribution - Pas d'Utilisation Commerciale - Pas de Modification 4.0 International. 


\title{
Regulierte Selbstregulierung. Historische Analysen hybrider Regelungsstrukturen
}

\author{
Einleitung
}

\author{
Peter Collin et Sabine Rudischhauser
}

1 Im Jahre 1881 gab der deutsche Verband der Dampfkesselüberwachungsvereine Vorschriften für die Prüfung von Dampfkesseln heraus. Geltung beanspruchen konnten sie an sich zunächst nur für die Unternehmen, die Mitglieder dieses Vereins waren. Einige Jahre später beschloss die preußische Verwaltung, in ihren gewerbepolizeilichen Bestimmungen auf diese Vorschriften zu verweisen, sie sollten also für alle Unternehmen gelten. Mit der Weiterentwicklung dieser technischen Regeln wurde die Deutsche Dampfkessel-Normen-Kommission beauftragt, die sich vor allem aus Vertretern verschiedener Industriebranchen zusammensetzte. Die aktuellen Fassungen wurden jeweils im Amtsblatt veröffentlicht. ${ }^{1}$

2 1931, nachdem in der Finanzkrise eine allgemeine Senkung der Zinsen immer dringlicher geworden war, bestimmte der deutsche Reichspräsident in einer Notverordnung die Zinssätze für eine Vielzahl von Bankgeschäften. Hinsichtlich anderer Bankgeschäfte setzte er den Finanzinstituten eine Frist, sich einvernehmlich auf eine Regelung zu einigen. ${ }^{2}$ Anfang 1932 schlossen schließlich die Zentralverbände von Banken, Sparkassen und Kreditinstituten ein entsprechendes Abkommen. Die Festlegung des Basiszinssatzes wurde darin einem "Zentralen Kreditausschuss" übertragen, der sich aus Vertretern der Finanzinstitute zusammensetzte. ${ }^{3}$

31936 brachte die Regierung der Volksfront in Frankreich ein Gesetz ein, das es dem Arbeitsminister erlaubte, Tarifverträge für allgemeinverbindlich zu erklären. In den Accords Matignon vom 7.Juni 1936 hatten sich die Arbeitgeber bereit erklärt, Tarifverhandlungen aufzunehmen. Das Gesetz vom 24.Juni 1936 etablierte ein Verfahren, wonach Tarifverträge durch paritätische Kommissionen ausgehandelt wurden, bestehend aus den Vertretern der für die jeweilige Branche »repräsentativsten« Gewerkschaften und Arbeitgeberverbände, die der Minister 
zusammenrief. Damit ein Tarifvertrag für allgemeinverbindlich erklärt werden konnte, musste er nicht nur die Höhe der Löhne festsetzen, sondern auch die Kündigungsfristen, die Wahl von Arbeiterdelegierten innerhalb der Betriebe und andere Gegenstände regeln, die bisher nicht gesetzlich geregelt worden waren. Bis Ende 1938 wurden 519 Tarifverträge, die auf diese Art und Weise zustande gekommen waren, für allgemeinverbindlich erklärt. ${ }^{4}$

4 So sehr sich die Beispiele auch hinsichtlich der Akteure und des Gegenstandsbezugs der erlassenen Normen unterscheiden, weisen sie jedoch eine Gemeinsamkeit auf: In jedem dieser Fälle ist es nicht allein der Staat, der regelsetzend tätig wird, sondern es sind nichtstaatliche Akteure. Allerdings beschränken sich diese nicht lediglich auf eine Koordination privater Interessen - selbst wenn dieses Motiv mehr oder weniger im Vordergrund gestanden haben mag. Vielmehr greift ihre Tätigkeit auch über in öffentliche Angelegenheiten: die allgemeine Sicherheit technischer Anlagen, die einheitliche Regelung von Arbeitsbedingungen und Löhnen oder die Herstellung allgemeiner Standards für Finanzgeschäfte. Und es handelt sich auch nicht um Regelungen, an denen der Staat unbeteiligt ist. Mal verschafft er diesen Normen erst allgemeine Geltung, mal erzwingt er die Schaffung neuen Rechts.

Derartige Konstellation lassen sich unter dem Begriff "regulierte Selbstregulierung" fassen. Absicht dieser Trivium-Nummer ist es zu veranschaulichen, wie die deutsche und französische Forschung dieses Phänomen historisch und theoretisch aufgearbeitet hat, auf welche Gegenstandsbereiche sie ihre Bemühungen konzentriert, wie sie sich dabei in ältere Forschungstraditionen einbettet und von welchen gesellschaftstheoretischen Vorannahmen sie sich leiten lässt.

Allerdings ist der Terminus "regulierte Selbstregulierung" selbst klärungsbedürftig. Denn erstens bettet er sich in eine komplexe Begriffsumgebung ein, in der veränderte Formen von Staatlichkeit und das veränderte Verhältnis von Staat und Gesellschaft in ihren verschiedenen Facetten erfasst werden. Und zweitens handelt es sich um eine moderne Begriffsschöpfung, deren Verwendbarkeit für historische Phänomene plausibel gemacht werden muss. Mit dem ersten Aspekt befassen sich die Erörterungen im Abschnitt I, mit dem zweiten in Abschnitt II.

\section{Begriffsverständnisse und Begriffsumgebungen}

\section{Regulierung und regulierte Selbstregulierung}

7 In der deutschen Diskussion ist zunächst einmal zwischen den Termini »Regulierung», "private Selbstregulierung" und "regulierte Selbstregulierung" zu unterscheiden. Der Begriff »Regulierung« entstand als eigenständiger Sammelbegriff für eine bestimmte Art von Normativität eigentlich erst in den 1990er Jahren, als mit der Organisationsprivatisierung ehemals staatlicher und monopolartig verfasster Infrastrukturen (Post, Eisenbahn, Telekommunikation) neue Regelungsbedürfnisse und vor allem die Notwendigkeit neuer Formen rechtlicher Gestaltung sichtbar wurden ${ }^{5}$ : Regulierung zielte auf die Herstellung wettbewerblicher Verhältnisse und auf die Hervorbringung marktwirtschaftlicher Innovationspotentiale und zugleich auf die Sicherung bestimmter Gemeinwohlbelange. Im Mittelpunkt stand allerdings die Frage, welche Normierungsanstrengungen von Seiten des Staates notwendig sind. In diesem Sinne verwendet auch die französische Rechtswissenschaft den Begriff $»$ Regulierung ${ }^{6}{ }^{6}$ 
Demgegenüber konzentrieren sich jene Überlegungen, die sich unter dem Begriff "private Selbstregulierung " zusammenfassen lassen und die ihre Herkunft vor allem im Privatrecht haben, auf Gestaltungsmodi nichtstaatlicher Akteure, auf Formen nichtstaatlicher Interessenorganisation mittels rechtlich verbindlicher Regelungen. ${ }^{7}$

Der Terminus "regulierte Selbstregulierung" gewann seine Konturen in Deutschland hingegen vor allem in einer öffentlich-rechtlichen Debatte ${ }^{8}$, die Konzepte zur Reform des Verwaltungsrechts entwickelte. Im Mittelpunkt stand dabei die Frage, welche Formen gesellschaftlicher Selbstorganisation in die Erfüllung öffentlicher Aufgaben eingebunden waren und wie man diese Arrangements von nichtstaatlicher Interessenverfolgung und staatlicher Beeinflussung angemessen begrifflichsystematisch erfassen konnte. Das Wortpaar "regulierte Selbstregulierung " hat sich hierfür inzwischen durchgesetzt, nachdem in den 1990er die maßgeblichen Debatten dazu stattgefunden hatten"; inzwischen ist "regulierte Selbstregulierung " zu einem anerkannten Schlüsselbegriff des Verwaltungsrechts geworden ${ }^{10}$ und wird als Analysekategorie zur Untersuchung verschiedener Verwaltungssektoren eingesetzt. ${ }^{11}$

In Frankreich hingegen hat im öffentlichen Recht keine vergleichbare Debatte stattgefunden, so dass sich bisher weder "regulierte Selbstregulierung" noch ein äquivalenter Begriff etablieren konnte. Ähnlichkeiten zum deutschen Begriffsverständnis scheint auf den ersten Blick die französische Théorie de la régulation sociale aufzuweisen, die der Soziologe Jean-Daniel Reynaud entwickelt hat. ${ }^{12}$ Doch handelt es sich bei den "règles sociales«, die Reynaud untersucht, keineswegs nur um rechtliche Normen. Die "régulation conjointe" entsteht aus der Interaktion jedweder, auch ausschließlich privater Akteure, z. B. innerhalb eines Unternehmens, während "regulierte Selbstregulierung" auf die Analyse von privat-staatlichen Regelungsstrukturen zur Erfüllung öffentlicher Aufgaben zielt. Allerdings haben sich französische Historiker seit den 1990er Jahren vermehrt mit solchen »intermediären« Organisationen befasst, die für die Wirtschaft und Arbeitswelt im 19. und 20. Jahrhundert Regeln setzten und durchsetzten. ${ }^{13}$ Deshalb stammen zwei der drei französischen Beiträge in diesem Band von Historikern. Sie geben auch Antworten auf die Frage, weshalb Frankreich »dasjenige Land der kontinentaleuropäischen Tradition [ist], in dem die Debatte über Selbstregulierung im öffentlichen Recht am wenigsten Anklang gefunden hat. $\ll^{14}$

\section{Verwandte Ansätze: Governance und Neokorporatismus}

10 Als Forschungsansatz für die Untersuchung privat-staatlicher Regelungsstrukturen kann »regulierte Selbstregulierung" allerdings keine Monopolstellung beanspruchen. Einen ähnlichen Fokus weist zunächst die Governance-Forschung auf. Allerdings ist auch der Begriff "Governance«, der in der deutschen wie der französischen juristischen, aber auch politologischen und soziologischen Debatte sehr wirkungsmächtig geworden ist ${ }^{15}$, klärungsbedürftig. Als normative Kategorie findet sich «Governance » als Recht auf " gute Verwaltung » in der Europäischen Charta der Grundrechte, Art. $41^{16}$, oder als "good governance « in den Richtlinien internationaler Organisationen verankert. Im hier behandelten Zusammenhang wird Governance aber nicht als normative Kategorie begriffen sondern als analytische Kategorie. In diesem Sinne lässt sich Governance verstehen als »Gesamtheit aller nebeneinander bestehenden Formen der kollektiven Regelung gesellschaftlicher Sachverhalte, von der 
institutionalisierten zivilgesellschaftlichen Selbstregelung über verschiedene Formen des Zusammenwirkens staatlicher und privater Akteure bis hin zu hoheitlichem Handeln staatlicher Akteure ${ }^{17}$ Die Verwandtschaft mit dem Begriff »regulierte Selbstregulierung« ergibt sich daraus, dass in beiden Fällen auf die Verwobenheit verschiedener normativer Schichten, die staatlichen oder nichtstaatlichen Ursprungs sein können, abgestellt wird..$^{18}$ Allerdings ist ein gravierender Unterschied zu beachten: »Regulierte Selbstregulierung" wird als Regelungsstruktur mit einer bestimmten funktionalen Ausrichtung verstanden. Sie dient (zumindest auch) der Erfüllung öffentlicher Aufgaben. Governance hingegen bleibt funktional indifferent. ${ }^{19}$

11 Verwandte Untersuchungsabsichten lassen sich auch einem Ansatz zuordnen, der sich am besten unter dem Begriff »Neokorporatismus« zusammenfassen lässt. Allerdings muss hierbei differenziert werden. "Korporatismus" kann zunächst als eher deskriptiver Sammelbegriff verstanden werden, der auf die Erfassung bestimmter zeitgenössischer ständestaatlicher Konzeptionen oder Gestaltungsformen zielt, die noch nach der Beseitigung der ständestaatlichen Ordnung bestanden oder entwickelt wurden. $^{20}$ Davon $\mathrm{zu}$ unterscheiden ist der Untersuchungsansatz des "Neokorporatismus«. Ausgehend von den wegweisenden Arbeiten Schmitters ${ }^{21}$ und in Abgrenzung von den traditionellen Pluralismustheorien ${ }^{22}$ identifizierte man neben Markt, Staat und Gemeinschaft ein weiteres Ordnungsmuster moderner Gesellschaften, das in seiner Funktionsfähigkeit auf die Existenz von Verbänden gründete, die gesellschaftliche Partialinteressen sammelten, koordinierten und vermittelten. ${ }^{23} \mathrm{Im}$ Unterschied zur hergebrachten Verbändeforschung, die diese Vereinigungen lediglich als auf den Staat einwirkende "pressure groups« wahrnahm, weitete man den Blick hier aus auf die wechselseitige Verwobenheit von Staat und Interessengruppen, die Einbeziehung nichtstaatlicher Verbandsmacht in staatliche Gestaltungsvorstellungen und die Abhängigkeit von der autoritätsvermittelnden Macht des Staates. Damit sind jedenfalls zu einem Gutteil - genau jene Funktionsmechanismen erfasst, die auch unter die Bezeichnung »regulierte Selbstregulierung ${ }^{24}$ subsumiert werden können.

\section{Regulierte Selbstregulierung in historischer Perspektive}

12 Obwohl der Begriff »regulierte Selbstregulierung" im Hinblick auf Gestaltungserfordernisse moderner Gemeinwesen entwickelt wurde, bietet er sich wegen seines hinreichend abstrakten Charakters auch als Analysekategorie für die Untersuchung weiter zurückliegender Zeitabschnitte an. Angezeigt scheint es aber, einen gewissen zeitlichen Schnitt zu machen: Erst im 19. Jahrhundert entstand eine organisatorische Basis für die - nicht auf ständischer Tradition, sondern auf bewusst organisierter Interessenwahrnehmung beruhende - Koordination von Partialbelangen: Berufsvereine und Branchenvereine und ihre Dachorganisationen, Assoziationen der Arbeiter und Bauern, Verbraucherinteressen bündelnde Organisationen, Unternehmenskonglomerate, halbamtliche Interessenvertretungen (Kammern) etc. Zugleich intervenierte die Gesetzgebung in immer weitere Gebiete des Wirtschaftslebens und übernahm zunehmend sozialpolitische Funktionen, mussten komplexe Verkehrs- und Versorgungsinfrastrukturen reguliert werden, vergrößerte sich der Beamtenapparat und entstanden neue Sonderbehörden. Zunehmende staatliche Regulierung stand vor neuen Herausforderungen, die in vielen Fällen nur 
durch Inanspruchnahme gesellschaftlicher Selbstorganisation bewältigt werden konnten.

Beide Prozesse - die Organisation gesellschaftlicher Akteure und die Entstehung des Interventionsstaates - nahmen aber in Deutschland und Frankreich einen ganz unterschiedlichen Verlauf. Während in Frankreich durch die Revolution alle ständischen Einrichtungen, Korporationen und Zünfte aufgelöst und verboten wurden, verloren sie in Deutschland im Zuge der Reformgesetzgebung nur ihren Zwangscharakter, bestanden aber vielerorts fort. ${ }^{25}$ Neue Organisationsformen konnten so auf den bestehenden aufbauen oder aus ihnen erwachsen, wie z. B. die Arbeitgeberverbände im Baugewerbe aus den Innungsverbänden. Darüber hinaus war der Zusammenschluss zu Vereinigungen in Deutschland, trotz aller Repression politischer Vereine und insb. von Arbeiterkoalitionen, grundsätzlich frei, eine Freiheit, die ab 1848 auch gesetzlich bzw. verfassungsrechtlich verankert wurde. In Frankreich dagegen wurden die rigorosen Strafbestimmungen des Code pénal gegen Vereinigungen erst 1901 abgeschafft, wichtiger noch: Antikorporatismus und Antipluralismus blieben für die Konzeption der öffentlichen Ordnung fundamental, über alle Regimewechsel hinweg. Die "jakobinische« Konzeption der "nation une et indivisible" verhinderte zwar nicht die Entfaltung eines Vereinswesen und die Entstehung von Interessenverbänden, erlaubte es jedoch nicht, die Frage zu diskutieren, "wie sich partikulare Interessen zum Gemeinwohl, wie sich organisierte gesellschaftliche Akteure zum Staat verhielten, ... weil die Existenz solcher Interessen und Akteure geleugnet wurde. $\aleph^{26}$ Die Formen regulierter Selbstregulierung waren in Frankreich weit stärker vom Bedarf des Staates an Information, Beratung und Instrumenten zur Durchsetzung gesetzlicher Maßnahmen bestimmt als vom Streben der Bürger nach Selbstregulierung. ${ }^{27}$

\section{Ambivalenzen und Paradoxien}

Regulierte Selbstregulierung ist ein Gestaltungsmodus, bei dem sich nicht nur "technische« Fragen nach der angemessenen Verteilung von Kompetenzen und nach effektiven Koordinationsmodi stellten. Vielmehr waren hiermit grundlegende Fragen nach der politischen Verfassung des Gemeinwesens aufgeworfen. Dabei zeigt sich, dass sich dieses Konzept $\mathrm{zu}$ den großen politischen Strömungen jener Zeit durchaus ambivalent verhielt.

Der Liberalismus war mit dem Versprechen staatsbürgerlicher Gleichheit und der universellen Herrschaft des Rechts angetreten. Die Etablierung von Sonderordnungen neben dem allgemeinen Gesetz, die an das Wiederaufkommen ständischer Sonderrechte denken ließ, widersprach dem. Andererseits gehörte der Selbstregulierungsgedanke, die Idee, dass gesellschaftliche Akteure ihre Angelegenheiten auch in kollektiver und normativer Form selbst regeln sollten, zum Markenkern liberalen Denkens in Deutschland. Auch innerhalb der liberalen Bewegung war es aber äußerst umstritten, inwiefern gesellschaftliche Akteure innerhalb vom Staat zur Verfügung gestellter Organisation, mit staatlicher Unterstützung oder gar subordiniert unter staatliche Zielvorgaben handeln sollten; die Auseinandersetzungen um die Organisation des Gewerbes in Handelskammern, um die Inanspruchnahme staatlicher Hilfe durch Kreditgenossenschaften oder um die Eingliederung von privaten Krankenkassen in die öffentliche Krankenversicherung zeugen davon. 
16 In Frankreich hingegen betonten Liberale die Rolle des Staates als Hüter staatsbürgerlicher Freiheit und Gleichheit weit stärker als das Recht gesellschaftlicher Akteure zu kollektivem Handeln. Dieser „Staatsliberalismus « ${ }^{28}$ war ein Erbe der Revolution, die die alten Sonderrechte radikal beseitigt hatte und hinter jeder Vereinigung gesellschaftlicher Kräfte eine Gefahr für die Einheit der Nation, hinter jedem Berufsverein ein Wiedererstehen der Zünfte gesehen hatte. Gegen dieses jakobinische Erbe konnte die liberale Forderung nach Vereinigungsfreiheit nur durchgesetzt werden, wenn der Staat durch enge Regulierungen jede Entstehung neuer Monopole und neuer Privilegien verhinderte und Vereinen wie Gewerkschaften finanzielle und politische Macht und Einfluss verwehrte. Formen der Selbstregulierung, die die Liberalen im II. Kaiserreich als Begrenzung autokratischer Herrschaft erstrebt hatten, mussten angesichts der demokratischen Verfassung der III. Republik neu begründet werden. Selbstregulierung war grundsätzlich nur legitim, soweit sie der Staat selbst organisierte.

17 Konservative zeigten sich dem Selbstregulierungsgedanken gegenüber offen, solange es sich um Formen handelte, die hergebrachte Vorrechte und Autoritäten achteten und sich in Vorstellungen einer »organischen« Verfassung der Gesellschaft einfügen ließen - akzeptiert wurde also eine Selbstregulierung, die hergebrachte Ordnungen reproduzierte. Eine Selbstregulierung hingegen, die diese Ordnungen nicht stabilisierte, sondern als bloße Interessenkoordination hergebrachte Schranken ignorierte und ihre Wirkmächtigkeit gerade nicht aus traditioneller Autorität gewann, stieß dagegen auf Ablehnung. In Deutschland vollzogen die Konservativen, eingebunden in das - natürliche - Bündnis von Monarchie und Regierungsapparat, auch deren politische Wendungen mit, wie sich vor allem gegen Ende des 19. Jahrhunderts zeigte. Konservative Politik bedeutete dann auch - gerade im Interesse der Wahrung der Stabilität politischer Herrschaft -, sich für neue Formen gesellschaftlicher Selbstregulierung aufgeschlossen zu zeigen, zumal wenn diese durch staatliche Macht eingehegt waren. ${ }^{29}$ Allerdings lag dann die konservative Sympathie in der Regel bei korporativ-ständisch verfassten Formen.

18 Sozialistisches Denken schließlich musste seine Konzepte im Spannungsverhältnis von chiliastischen Erwartungen und tagespolitischer Pragmatik entwickeln. Dies trat gleich in zweierlei Weise $\mathrm{zu}$ Tage. Erstens distanzierte man sich von Vorstellungen bürgerlicher Selbstregulierung, gestaltete die soziale Selbsthilfe und die politische Organisation der Arbeiterschaft aber in genau diesen bürgerlichen Formen, dem Verein und der Genossenschaft, und orientierte sich dabei auch an bürgerlichen Konventionen von Willensbildung und Entscheidungsvermittlung. ${ }^{30}$ Zweitens bestimmte eine grundsätzliche Ablehnung der herrschenden Staatsmacht das sozialistische Denken. Dort aber, wo Sozialisten bestehende Einflussmöglichkeiten wahrnehmen wollten, nahmen sie nicht nur an Wahlen teil und besetzten Wahlämter auf Reichs-, Landes- und Kommunalebene; sie übernahmen auch Funktionen in halbamtlichen Einrichtungen wie Krankenkassen oder Gewerbegerichten. Gerade im Bereich sozialpolitischer Selbstregulierung wurden Repräsentanten der sozialistischen Bewegungen zur tragenden Kraft. 


\section{Spielarten}

Bisherige Untersuchungen zu regulierter Selbstregulierung im 19. und frühen 20. Jahrhundert, die sich auf Deutschland konzentrierten ${ }^{31}$, aber sich auch auf andere westund mitteleuropäische Länder sowie die USA erstreckten ${ }^{32}$, haben eine außerordentliche Vielfalt von Erscheinungsformen zu Tage gefördert (wobei der Schwerpunkt auf dem Gebiet der Wirtschaft und der Sozialpolitik lag ${ }^{33}$ ). Diese Mannigfaltigkeit lässt sich - je nach disziplinärem Untersuchungsinteresse - in unterschiedlicher Weise darstellen. Die folgenden Ausführungen beschränken sich auf zwei Ordnungsversuche: erstens auf eine Darstellung, die sich an funktionalen Aspekten orientiert, zweitens auf eine Systematisierung, die Formen der Selbstregulierung hinsichtlich ihrer mehr oder weniger ausgeprägten Staatsnähe unterscheidet. Geschildert werden in erster Linie deutsche Verhältnisse, nur punktuell ergänzt um französische Beispiele, weil eine solche Systematisierung der Formen regulierter Selbstregulierung für Frankreich bisher noch ein Forschungsdesiderat darstellt. ${ }^{34}$

\section{a) Funktionale Ausrichtungen}

\section{aa) Selbstregulierung als Normsetzung}

Unter funktionalen Gesichtspunkten lassen sich normsetzende, normdurchsetzende und justizielle spielarten regulierter selbstregulierung unterscheiden. Aus unterschiedlichen Gründen steht die Normsetzung im Vordergrund des Interesses. Dies liegt einmal daran, dass der Begriff Selbst»regulierung " von seiner Wortbedeutung her auf Regelsetzung verweist, aber auch daran, dass Rechtssetzung wegen ihrer kollektiv bindenden Wirkung am ehesten zu den staatlichen Prärogativen gezählt wird und die Ausübung dieser Funktion durch nichtstaatliche Akteure wegen ihrer besonderen Begründungsbedürftigkeit am ehesten rechtsgeschichtliches, aber auch politikgeschichtliches Interesse weckt.

21 Dabei muss allerdings zwischen verschiedenen Normschichten differenziert werden. Der Funktion staatlicher Rechtssetzung am nächsten kam es, wenn nichtstaatlichen Einrichtungen die Aufgabe übertragen war, Regeln zu erlassen, die als staatliches Recht eingestuft wurden. Ein solcher Fall ist die Normsetzung durch den Reichskohlenverband, der 1919 im Zuge der Wirtschaftsdemokratisierung errichtet worden war und Regelungen für den Kohlenhandel $\mathrm{zu}$ erlassen hatte. ${ }^{35}$ Der Reichskohlenverband war eine privatrechtliche Gesellschaft, der sich aus den privaten Syndikaten der Kohlenwirtschaft zusammensetzte; gleichwohl wurden seine Bestimmungen als Rechtsverordnungen eingestuft. ${ }^{36} \mathrm{Im}$ Ganzen gesehen handelte es sich dabei allerdings um einen Ausnahmefall. Der gängige Fall war der Erlass von Satzungen. Soweit es sich um Satzungen öffentlich-rechtlich verfasster Organisationen nichtstaatlicher Akteure (z. B. Krankenkassen, Handelskammern oder Wassergenossenschaften) handelte, wurden deren Bestimmungen als Rechtsnormen qualifiziert; der Rechtscharakter von Satzungen privatrechtlicher Organisationen (z. B. Vereine) war hingegen umstritten. Allerdings entfalteten Satzungen in den allermeisten Fällen ${ }^{37}$ nur Bindungswirkung innerhalb des Mitgliederkreises dieser Verbände. Weiter hat man es mit interverbandlichen Vereinbarungen zu tun. Soweit diese Vereinbarungen von Dachverbänden geschlossen wurden, normierten sie im 
Regelfall auf nationaler Ebene weite Bereiche der wirtschaftlichen Interaktion innerhalb eines bestimmten Sektors. Derartiges lässt z. B. im Finanzsektor beobachten, dessen Wettbewerbsverhältnisse durch Vereinbarungen zwischen den Dachverbänden der Sparkassen, Kreditgenossenschaften und Banken geregelt wurden, oder im Bereich der gesetzlichen Krankenversicherung, wo die Konditionen ärztlicher Behandlung eine Regelung durch Verträge zwischen den Zentralverbänden von Krankenkassen und Ärzten erfuhren. In Frankreich legten Tarifverträge zwischen Arbeitgeberverbänden und Gewerkschaften die lokalen Arbeitsbedingungen für das ganze Baugewerbe fest, weil viele französische Städte die tarifvertraglichen Löhne in ihre offiziellen Preisverzeichnisse und Lastenhefte übernahmen. ${ }^{38}$ Schließlich lassen sich weitere zahlreiche Formen der Normsetzung konstatieren, die aufgrund ihrer Verschiedenartigkeit nicht ohne weiteres gruppenmäßig zusammengefasst werden können: technische Standardsetzung durch Ingenieurs- oder Unternehmensverbände ${ }^{39}$, Kodifikationen kaufmännischer Usancen oder Geschäftsbedingungen durch Handelskammern ${ }^{40}$, ebenfalls durch Handelskammern verfasste Schiedsgerichtsreglements ${ }^{41}$, Beförderungsreglements, die von Dachverbänden der Eisenbahngesellschaften ausgearbeitet wurden $^{42}$, sowie Kodifikationen der arbeitsrechtlichen Usancen durch Conseils de prud'hommes in Frankreich. ${ }^{43}$

\section{bb) Selbstregulierung als funktionales Äquivalent administrativer Gewalt}

Neben diese normsetzende Selbstregulierung treten weitere Formen der Ausübung von Autorität - die in gewisser Weise als funktionales Äquivalent staatlicher Autorität angesehen werden kann. Hierzu zählen erstens administrative, normdurchsetzende Modi. Zunächst hat man es damit zu tun, dass in einem Gutteil der oben genannten Fälle, in denen nichtstaatliche Akteure Regelungen erließen, diese auch tätig wurden, um diese Regeln durchzusetzen. Allerdings war dies noch nicht gleichbedeutend mit der Ausübung von Autorität, die amtlicher Gewalt gleichkam. Derartige Autorität entstand im Regelfall erst durch staatliche Delegation. Diese konnte sich soweit erstrecken, dass intermediären Verbänden ein ganzer Verwaltungszweig überantwortet wurde. $\mathrm{Zu}$ weiten Teilen geschah dies im Bereich der Sozialversicherung. Die Krankenkassen verantworteten $\mathrm{zu}$ einem Gutteil die Umsetzungen des Krankenversicherungsgesetzes ${ }^{44}$, die Berufsgenossenschaften waren zuständig für die Ausführungen der Bestimmungen des Unfallversicherungsgesetzes ${ }^{45}$ auch wenn natürlich staatlichen Behörden die Kompetenz für bestimmte Einzelaspekte und vor allem für die Staatsaufsicht verblieb. Dies war aber nicht der Regelfall. Typisch war eher die Überweisung bestimmter Einzelbefugnisse, so die Übernahme von Eisenbahnpolizeiaufgaben sowie eines Teils des Enteignungsverfahrens durch Eisenbahngesellschaften ${ }^{46}$, die Organisation der Ausbildung und Prüfung im Handwerk durch die Handwerkskammern ${ }^{47}$ oder die Überwachung gefährlicher technischer Anlagen durch private Überwachungsvereine ${ }^{48}$.

\section{cc) Justizielle Selbstregulierung}

Schließlich ist auf eine in der Forschung bisher weitgehend vernachlässigte Spielart von Selbstregulierung aufmerksam zu machen: die justizielle Selbstregulierung. Die Rechtsgeschichte hat sich bisher allzu sehr auf die Entwicklung der staatlichen Justiz konzentriert. Jedoch entwickelten sich gerade im hier interessierenden Zeitraum zahlreiche Formen justizieller Konfliktlösung außerhalb der ordentlichen 
Gerichtsbarkeit. Auch diese wiesen eine außerordentliche Vielgestaltigkeit auf. Gemeinsames Merkmal derartiger Einrichtungen ist, dass in ihnen Repräsentanten nichtstaatlicher Partialbelange über Konflikte innerhalb gesellschaftlicher Teilbereiche entschieden und sich jedenfalls teilweise von sektorspezifischen Entscheidungsrationalitäten leiten ließen, die im staatlichen Gesetz nur unvollkommenen Ausdruck gefunden hatten.

Derartige Einrichtungen existierten in unterschiedlichen organisationalen Bezügen. In die staatliche Gerichtsbarkeit eingegliedert waren die Conseils de prud'hommes und die Gewerbegerichte, die Streitigkeiten zwischen Arbeitgebern und Arbeitnehmern, sowie die Kaufmannsgerichte, die Streitigkeiten zwischen kaufmännischen Unternehmern und ihren Angestellten entschieden..$^{49}$ Nicht integriert in das staatliche Justizsystem, aber öffentlich-rechtlichen Institutionen angegliedert waren die Ehrengerichte bei den berufsständischen Kammern. ${ }^{50}$ Daneben hat man es mit einer außerordentlichen Vielfalt von Einrichtungen zu tun, die unter solchen Bezeichnungen wie Schiedsgericht $t^{51}$, Einigungsamt oder Schiedsstelle firmierten. Sie entschieden z. B. über interorganisationale Konflikte ${ }^{52}$, über sozialversicherungsrechtliche Streitigkeiten ${ }^{53}$ oder (oft nach »billigem Ermessen«) über die Nutzung oder Verteilung knapper Ressourcen ${ }^{54}$; letztere waren vor allem in Krieg- und Krisenzeiten errichtet worden und hatten teilweise nur eine kurze Lebensdauer. Gemeinsam war ihnen, dass in ihnen nicht staatliche Richter judizierten, sondern in der Regel paritätisch vertretene Repräsentanten der jeweils betroffenen sozialen Gruppen - wenn auch teilweise unter Vorsitz eines Juristen, der vor allem einen geordneten Verfahrensgang sicherstellen sollte.

\section{b) Staatsnähe und Staatsferne}

Die Anbindung derartiger Selbstregulierungsforen an staatlich definierte und staatlich zu garantierende Gemeinwohlbelange wurde durch unterschiedliche Mechanismen sichergestellt. Wie staatsnah oder staatsfern solche Arrangements verfasst waren, lässt sich nach verschiedenen Kriterien bestimmen.

\section{aa) Private und öffentliche Organisationsrechtsformen}

Nichtstaatliche Akteure konnten in öffentlich-rechtlichen oder in privatrechtlichen Verbänden organisiert sein. Dabei ist eine Vorbemerkung notwendig. In der heutigen öffentlich-rechtlichen Debatte schließt man öffentlich-rechtliche Organisationsformen aus dem Kreis der Träger regulierter Selbstregulierung aus. ${ }^{55}$ Derartige Einrichtungen werden zur "mittelbaren Staatsverwaltung" und damit zum "Staat«, nicht zur "Gesellschaft" gezählt. Unter den Bedingungen des 19. und frühen 20. Jahrhunderts erscheint eine derartige Einstufung nicht angemessen. Obgleich in das staatliche Organisationsgehäuse integriert, handelte es sich doch um Vereinigungen nichtstaatlicher Akteure. Ihre innere Verfassung und die Art der Entscheidungsfindung entsprach eher der des Vereins als einer staatlichen Behörde. Die typische deutsche Organisationsrechtsform war die der öffentlich-rechtlichen Körperschaft. Hierunter fielen z. B. Wirtschafts- und Berufskammern, Krankenkassen, Berufsgenossenschaften der Unfallversicherung oder Wassergenossenschaften. Gleichwohl war die öffentlichrechtliche Körperschaft die Organisationsform mit der größten Staatsnähe. Sie stand unter Staatsaufsicht und zahlreiche ihrer Entscheidungen standen unter 
Genehmigungsvorbehalt. Allerdings war die Staatsaufsicht meist auf die Rechtsaufsicht beschränkt, ein Eingriffsrecht des Staates bestand somit nur, wenn die betreffende Körperschaft rechtsfehlerhaft gehandelt hatte.

Selbstregulierungsakteure konnten aber auch in privatrechtsförmiger Gestalt organisiert sein. Dies war typisch für Frankreich, wo entsprechende Verbände dann in Form einer bürgerlich-rechtlichen Gesellschaft (société), als Verein (association) oder als Genossenschaft (mutuelle) wirkten. Zur Wahrnehmung bestimmter Aufgaben, die im öffentlichen Interesse lagen, wie z. B. Bodenverbesserungen oder Flutschutz, stellte der französische Gesetzgeber besondere privatrechtliche Formen zur Verfügung, z.B. Sociétés de crédit foncier oder Associations syndicales.

Auch in Deutschland findet man privatrechtlich organisierte Selbstregulierungsverbände - also an sich als staatsfern einzustufende Organisationsformen, da weder ihre Bildung noch ihre Satzungsgebung staatlicher Genehmigung bedurften ${ }^{56}$ und sie auch nicht der Staatsaufsicht unterlagen. Allerdings bestanden auch hier durchaus mannigfaltige Steuerungsmechanismen: die Vergabe staatlicher Subventionen konnte von der Erfüllung bestimmter Zwecke abhängig gemacht werden. Vereinsvorstände konnten mit Vertretern der staatlichen Amtsgewalt besetzt sein ${ }^{57}$, per Gesetz konnte die Ausführung bestimmter Aufgaben zur Pflicht gemacht werden. Dies war auch in Frankreich der Fall. So wurden im Kaiserreich die Vorsitzenden der Sociétés de secours mutuels approuvées von der Regierung ernannt, und in der III. Republik erhielten Gewerkschaften staatliche Subventionen, wenn sie Fortbildungskurse und Arbeitsvermittlungsbureaus einrichteten. Auch in diesem Bereich lassen sich Abstufungen feststellen, die von nahezu vollständiger staatlicher Steuerungsabstinenz bis zu faktisch weitgehender staatlicher Beherrschung reichten.

\section{bb) Freiwilligkeit und Zwang}

Regulierte Selbstregulierung speiste sich zum einen aus dem Interesse der beteiligten Akteure, ihre Angelegenheiten derart $\mathrm{zu}$ koordinieren, dass die Reproduktionsbedingungen des einzelnen Beteiligten zumindest hinreichend gesichert wurden, jedenfalls dann, wenn die Marktbedingungen dies nahelegten ${ }^{58}$ In dieser Hinsicht ist von einer intrinsischen Motivation auszugehen und von einem hohen Maße an Freiwilligkeit. Da regulierte Selbstregulierung allerdings in öffentliche Angelegenheiten übergriff, kann man auf der anderen Seite von einem vitalen Bedürfnis des Staates ausgehen, derartige Regelungsarrangements ins Leben zu rufen oder auch zu untersagen, wenn darin eine Gefährdung des Gemeinwohls gesehen wird.

Tatsächlich hat man es mit Gemengelagen zu tun, die zwischen Freiwilligkeit und Zwang oszillierten. So bildeten sich technische Prüfvereine zunächst aus rein privater Initiative und in Konkurrenz zu staatlichen Überwachungsbehörden heraus. ${ }^{59}$ Auf der anderen Seite kann man nach dem Ende des Ersten Weltkriegs beobachten, wie der Staat Konglomerate der Grundstoffindustrie, die sich ihren eigenen Regelungskorpus schufen, per Gesetz erzwang. ${ }^{60}$ Aber meist hat man es damit zu tun, dass private Freiwilligkeit und staatlicher Zwang an irgendeiner Stelle zusammentrafen. Staatliche Organisationszwänge konnten auf langgehegte Organisationswünsche wirtschaftlicher Akteure treffen, wie z. B. bei den Handwerkskammern. ${ }^{61}$ Staatlicher Druck, bestimmte wirtschaftliche Interaktionen endlich privat $\mathrm{zu}$ regeln, konnte Verständigungsblockaden privater Verbände endlich überwinden, wie der 
Ausgestaltung der Beziehungen zwischen Ärzten und Krankenkassen. ${ }^{62}$ Freiwilligkeit bei der Bildung neuer Organisationsformen konnte schließlich auch von dem Wunsch getragen sein, an staatliche Unterstützung zu gelangen. Staatlicher Zwang und private Freiwilligkeit konnten somit in einer Weise verschmelzen, die von den äußerlichen Formen der jeweiligen Regelung nur unvollständig wiedergegeben wird.

\section{cc) Mittelbare oder unmittelbare Selbstregulierung}

Regulierte Selbstregulierung kann zum einen unmittelbar stattfinden. Das bedeutet, dass die nichtstaatlichen Akteure ihre Regeln selbst erlassen oder Konflikte durch eigene Instanzen, in denen keine staatlichen Repräsentanten vertreten sind, selbst entscheiden. Der Staat ist hier jedenfalls als unmittelbarer Entscheidungsakteur nicht beteiligt. Als mittelbare Selbstregulierung hingegen kann es angesehen werden, wenn die nichtstaatliche Seite ihre Regelungsvorstellungen im Rahmen staatlicher Entscheidungsprozesse zur Geltung bringen kann, sie also formell nicht Entscheidungsinstanz ist, aber auf die Entscheidungsfindung Einfluss nehmen kann. Hierfür boten die zahlreichen Beiräte, die vermehrt seit den 1880er Jahren in Deutschland und Frankreich entstanden, ein Forum. In diesen Beiräten (conseils supérieurs), die Ministerien oder zentralen Verwaltungsorganen beigeordnet waren, waren im Regelfall Repräsentanten der betroffenen gesellschaftlichen Gruppen vertreten. Allerdings kann man nicht davon ausgehen, dass nichtstaatliche Interessen unmittelbar in staatliche Regelungen Eingang fanden. Die Stimme der gesellschaftlichen Interessenvertreter war oft nur eine in einem mehrstufigen und komplexen Entscheidungsfindungsprozess mit vielen Beteiligten. Zudem dienten Beiräte nicht selten nur als Partizipation simulierendes Verfahrenselement in einem Prozedere, in dem sich die staatliche Bürokratie nicht die Entscheidungsprärogative aus den Händen nehmen ließ.63

Gewissermaßen als Mischform lässt sich das einstufen, was man Ko-Regulierung nennen kann. Darunter sollen hier solche Formen der Entscheidungsfindung verstanden werden, in denen staatliche und nichtstaatliche Repräsentanten als gleichberechtigte Entscheidungsakteure auftraten (womit noch keine Aussage über die jeweiligen Stimmverhältnisse getroffen ist). In das herrschende staatsrechtliche Verständnis passten solche kondominialen Gremien an sich nicht. Aber sie finden sich überall in jenen Einrichtungen, für die eine Besetzung sowohl mit staatlichen als auch mit Vertretern der betroffenen sozialen Gruppen vorgeschrieben war, vor allem in Organisationen der Kriegs- und der Gemeinwirtschaft ${ }^{64}$ und im Sektor der Sozialpolitik.

\section{Zu den einzelnen Beiträgen}

Bei der Auswahl der deutschen Beiträge waren verschiedene Kriterien leitend. Zum einen sollten sie einen ersten, wenngleich möglichst breiten Überblick über das historische Phänomen selbst bieten. Zum anderen sollte sich in ihnen die Entwicklung der rechts- bzw. verfassungsgeschichtlichen Forschung in Bezug auf Theoriegeneigtheiten und gesellschaftskonzeptionelle Vorstellungen spiegeln. Dies erklärt auch den Rückgriff auf schon weiter zurückliegende Publikationen.

Den Anfang bei den deutschen Aufsätzen macht ein Beitrag von Ulrich Scheuner, der einerseits einen instruktiven Überblick über die Entwicklung des Verbandswesens vom 
ausgehenden "absolutistischen" Zeitalter bis zum Beginn der staatsinterventionistischen Zeit in den 1870er Jahren und dessen zeitgenössische theoretische Reflexion vorlegt, sich also gewissermaßen mit der »Inkubationsphase« regulierter Selbstregulierung befasst. Andererseits spiegelt sich im Beitrag Scheuners der Abschluss der Debatte über das Verbandswesen, die die Bundesrepublik besonders in ihren ersten beiden Jahrzehnten intensiv beschäftigt hatte. ${ }^{65}$ Im Ergebnis hatte man sich mit den auf staatliche Entscheidungen Einfluss nehmenden Verbänden ausgesöhnt und damit auch geöffnet für pluralistische Gesellschaftsmodelle. Es sollte sich aber letztlich um einen staatlich überwölbten Pluralismus handeln, wie auch bei Scheuner deutlich wird, der in seinen Abschlussbemerkungen vor einer Vereinnahmung des Staates durch Verbandsinteressen warnt.

Letztlich zeigt sich damit aber immer noch eine Prägung der Diskussion, die einseitig die Einwirkung der Verbände auf den Staat in den Blick nimmt. Eine gewissermaßen umgekehrte Perspektive präsentiert der Beitrag von Michael Stolleis, der zeitlich da ansetzt, wo Scheuner aufgehört hat. Mit der Entstehung des Interventionsstaates war für ihn die Instrumentalisierung nichtstaatlicher Interessenverfolgung für staatliche Zwecke verbunden. Indem Stolleis den Blick auf die neu entstandenen hybriden privatstaatlichen Regelungsstrukturen lenkt, plädiert er gleichzeitig für eine stärkere Hinwendung der Rechtsgeschichte zu normativen Phänomenen, welche bisher von der »klassischen« Geschichte des Privat- bzw. des öffentlichen Rechts nur unzureichend erfasst worden waren.

Eine wiederum andere Herangehensweise wird im Aufsatz von Dieter Grimm sichtbar. Erschienen ist er in einem Sammelband, in dem die gegenwartsbezogene Debatte zu regulierter Selbstregulierung zusammenfand. Dem Beitrag von Grimm kam erkennbar die Aufgabe $\mathrm{zu}$, eine historische Verortung vorzunehmen. Anknüpfend an frühere verfassungs- und rechtsgeschichtliche Modellierungen ${ }^{66}$ entwickelt Grimm ein historisches Stufenmodell, in dem auf das umfassende Geltung beanspruchende staatliche Regulierungsregime des Absolutismus das Zeitalter der bürgerlichen Gesellschaft folgt, in welchem staatliche Regulierung durch Gesetz und bürgerliche Selbstkoordinierung durch Vertrag in relativ getrennten Sphären stattfinden. Mit dem Aufkommen des Interventionsstaates erweitert sich der staatliche Regulierungsraum wieder, was aber zunehmend Dysfunktionalitäten hervorbringt. Regulierte Selbstregulierung erscheint dann als - wenngleich nicht unproblematische intelligente Lösung.

Allerdings impliziert dies erstens wiederum die Vorstellung getrennter Sphären von Staat und Gesellschaft, die sich erst in neuerer Zeit aufgelöst haben und zweitens damit die historische Nichtexistenz regulierter Selbstregulierung. Demgegenüber versucht der Beitrag von Peter Collin - gerade auch anknüpfend an die gegenwartsbezogenen Kategorisierungen der öffentlich-rechtlichen Diskussion - das Ordnungsmodell der regulierten Selbstregulierung auch als rechtsgeschichtlich ertragreiche Kategorie einzuführen, indem er sondiert, wie Organisationsformen und Regelungsarrangements, die sich im 19. Jahrhundert herausgebildet haben, einerseits privatkollektive Belange organisierten, andererseits staatlichen Zielvorstellungen unterworfen wurden. Zugleich soll damit auch die Interaktion von privatrechtlichen und öffentlichrechtlichen Formen schon in dieser Phase der Rechtsentwicklung verdeutlicht werden.

Dabei konzentriert sich dieser Beitrag noch auf die Anfangsphase, gewissermaßen auf die frühe Kindheit regulierter Selbstregulierung. Zeitlich daran anschließen lässt sich 
der Aufsatz von Gerd Bender, in dem aber auch noch andere Gesichtspunkte zum Tragen kommen, die für eine rechtshistorische Diskussion um regulierte Selbstregulierung von Bedeutung sind. Erstens konzentriert er sich auf ein Referenzgebiet regulierter Selbstregulierung, nämlich das Arbeitsrecht. Zweitens führt er zwei Debattenströme zusammen, die auch der rechtshistorischen Forschung wesentliche Impulse liefern können, nämlich die Systemtheorie, die wegen der Anerkennung von funktionalen teilsystemischen Rationalitäten schon immer für die Anerkennung von nichtstaatlicher Selbstregulierung offen war, und die Korporatismustheorie, die - wie oben bereits ausgeführt - Analysemuster für die Erfassung privat-staatlicher Koordination zur Verfügung stellt.

Die französischen Beiträge unterstreichen die große Bedeutung regulierter Selbstregulierung für die französische Geschichte des 19. und 20. Jahrhunderts. Wie eingangs angedeutet, wurde dieses Phänomen bisher vor allem von Sozial- und Wirtschaftshistorikern sowie Politikwissenschaftlern untersucht, die Institutionen, insbesondere solche zur Regulierung der Wirtschaft, in den Focus ihrer Forschungen stellen. Dagegen gibt es nur wenige Beiträge von Rechtswissenschaftlern zu dieser Frage, die von Verfassungs- und Verwaltungsrechtlern kaum diskutiert wird.

Für das französische Arbeitsrecht aber ist, wie Alain Supiots Beitrag deutlich macht, das Zusammenwirken der Arbeitsmarktparteien und des Staates in einer Vielzahl von Kommissionen, Beiräten und Institutionen prägend. Indem Supiot Durkheims Konzeption eines Korporatismus zur Analyse des aktuellen Arbeitsrechtes nutzt, zeigt er zugleich, dass der Ruf nach Normsetzung durch nichtstaatliche Akteure der Kritik am Interventionsstaat entspringt. Hielt Durkheim solche Akteure für unentbehrlich, um die Entstehung eines »hypertrophen « Staates zu vermeiden, so erlaubt die Selbstregulierung der kollektiven Akteure in den 1980er Jahren dem Staat, sich aus der Regulierung der Arbeitswelt ein Stück weit zurückzuziehen, ohne sie dem freien Spiel der Kräfte bzw. dem Unternehmer allein zu überantworten. Supiot zieht so die Verbindung zur Debatte über die "Steuerungsproblematik moderner Industriegesellschaften", die in Deutschland bezeichnenderweise unter dem Titel "Grenzen des Rechts« geführt wurde. Wie Dieter Grimm im oben vorgestellten Beitrag sieht auch Supiot regulierte Selbstregulierung als »intelligentere« Alternative zur Deregulierung.

41 Supiot weist in diesem Artikel von 1987 auch darauf hin, dass Durkheims Bemühungen, neue, vom Korporatismus des Ancien régime grundverschiedene Formen der Selbstregulierung, basierend auf staatsbürgerlicher Gleichheit und Herrschaft des Gesetzes, zu begründen, auf Unverständnis stießen. Hieran knüpft der Beitrag von Steven L. Kaplan und Philippe Minard 17 Jahre später an. Die Autoren arbeiten nicht nur die Besonderheiten der Stellung der Verbände in Frankreich heraus und geben einen Überblick über die vielfältigen historischen Arbeiten, die die Praxis des Zusammenwirkens zwischen staatlicher Verwaltung und gesellschaftlichen Organisationen und den Diskurs darüber erforscht haben. Sie erklären vor allem die Ursachen für die von Durkheim bis heute zu beobachtende Schwierigkeit, organisierter Interessenvertretung eine legitime Rolle in der französischen politischen Demokratie zuzuschreiben.

In ihrer Rezension greift Claire Lemercier die Thesen von Kaplan und Minard auf und stellt die Forschungsergebnisse der Sozial- und Wirtschaftshistoriker den Arbeiten Rosanvallons gegenüber, des wichtigsten Vertreters einer neuen Politikgeschichte. 
Beide Forschungsrichtungen betonen, so Lemercier, die Kluft zwischen einem »jakobinischen« Denken und der faktischen Existenz und Rolle einer Vielfalt von gesellschaftlichen Institutionen und Organisationen. Rosanvallon ebenso wie Kaplan und Minard und die Autoren des von ihnen herausgegebenen Sammelbandes gelangen zu einem neuen Bild des Verhältnisses von Staat und Gesellschaft in Frankreich, indem sie sowohl die praktische Bedeutung von Formen regulierter Selbstregulierung als auch das Gewicht einer Definition des Gemeinwohls berücksichtigen, die eine Mitwirkung widerstreitender, partikularer Interessenverbände ausschloss. Lemerciers benennt Forschungsdesiderate, darunter die schon 1987 von Supiot angemahnte Typologie der Formen und Funktionen kollektiver Organisationen, die an öffentlichen Belangen gestaltend mitwirken, und eine stärkere Berücksichtigung der rechtlichen Dimension, um Formen des Zusammenwirkens von staatlicher Verwaltung und gesellschaftlichen Organisationen genauer $\mathrm{zu}$ beschreiben und schließlich auch den Vergleich mit anderen Staaten zu ermöglichen. Sie skizziert so, ohne den Begriff zu verwenden, ein Forschungsprogramm zur regulierten Selbstregulierung in Frankreich.

\section{BIBLIOGRAPHIE}

Ayass, W. (2012): »Regulierte Selbstregulierung in den Berufsgenossenschaften der gesetzlichen Unfallversicherung «, in: Collin. P. / Bender, G. / Ruppert, S. / Seckelmann, M. / Stolleis, M. (Hg.): Regulierte Selbstregulierung im frühen Interventions- und Sozialstaat, Frankfurt/M., S. 123-143.

Ayass, W. (2016): »Die Rechtsprechung in der Sozialversicherung bis zur Reichsversicherungsordnung. Beteiligte, Institutionen, Verfahren«, in: Collin, P. (Hg.): Justice without the State within the State, Frankfurt/M.

Bachmann, G. (2006): Private Ordnung. Grundlagen ziviler Regelsetzung, Tübingen.

Bosch, D. (2007): Die »Regulierte Selbstregulierung« im Jugendmedienschutz-Staatsvertrag, Frankfurt/ M. u.a.

Botzem, S. u.a. (Hg.) (2009): Governance als Prozess, Baden-Baden.

Braibant, G. (2001) : La Charte des droits fondamentaux de l'Union européenne. Témoignage et commentaires, Paris.

Brand, J. (1990/2002/2008): Untersuchungen zur Entstehung der Arbeitsgerichtsbarkeit in Deutschland, Tbd. 1-3, Pfaffenweiler / Frankfurt/M.

Buck-Heeb, P. / Dieckmann, A. (2010): Selbstregulierung im Privatrecht, Tübingen.

Chatriot, A. / Lemercier, C. (2008): »Une histoire des pratiques consultatives de l’Etat «, in: Offerle, M. / Rousso, H. (Hrsg.): La fabrique interdisciplinaire: histoire et science politique, Rennes, S. 191-203.

Collin. P. / Bender, G. / Ruppert, S. / Seckelmann, M. / Stolleis, M. (Hg.) (2011): Selbstregulierung im 19. Jahrhundert - zwischen Autonomie und staatlichen Steuerungsansprüchen, Frankfurt/M.

Collin. P. / Bender, G. / Ruppert, S. / Seckelmann, M. / Stolleis, M. (Hg.) (2012): Regulierte Selbstregulierung im frühen Interventions- und Sozialstaat, Frankfurt/M. 
Collin. P. / Bender, G. / Ruppert, S. / Seckelmann, M. / Stolleis, M. (Hg.) (2014): Regulierte Selbstregulierung in der westlichen Welt des späten 19. und frühen 20. Jahrhunderts, Frankfurt/M.

Darnaculleta i Gardella, M. M. (2014): »Wahrnehmungen >regulierter Selbstregulierung in der angelsächsischen und kontinentaleuropäischen Literatur (seit den 90er Jahren des 20. Jahrhunderts)«, in: Collin. P. / Bender, G. / Ruppert, S. / Seckelmann, M. / Stolleis, M. (Hg.): Regulierte Selbstregulierung in der westlichen Welt des späten 19. und frühen 20. Jahrhunderts, Frankfurt/M., S. 57-73.

Deparade, J. (1927): Die Kodifikation von Handelsgebräuchen durch die Industrie- und Handelskammern und ihre Bedeutung für den Handelsverkehr und die Rechtsentwicklung, Magdeburg.

Di Fabio, U. (1997): »Verwaltung und Verwaltungsrecht zwischen gesellschaftlicher Selbstregulierung und staatlicher Steuerung «, Veröffentlichungen der Vereinigung der Deutschen Staatsrechtslehrer, 56, Berlin / New York, S. 235-282.

Didry, C. (1998) : »La nouvelle jeunesse des conventions collectives: la loi du 24 juin 1936«, in: Le Crom, J.-P. (Hg.): Deux siècles de droit du travail. L'histoire par les lois, Paris, S. 129-140.

Fahrmeir, A. (2011): »Regulierte Selbstregulierung in Großbritannien«, in: Collin. P. / Bender, G. / Ruppert, S. / Seckelmann, M. / Stolleis, M. (Hg.): Selbstregulierung im 19. Jahrhundert - zwischen Autonomie und staatlichen Steuerungsansprüchen, Frankfurt/M., S. 113-126.

Frenzel, S. (2007): Stromhandel und staatliche Ordnungspolitik, Berlin.

Frison-Roche, M.A. (Hg.) (2004): Les régulations économiques : légitimité et efficacité, coll. »Droit et Economie de la Régulation«, Bd. 1, Paris.

Frison-Roche, M.A. (Hg.) (2005): Les risques de régulation, Bd. 3, Paris.

Führer, K.-C. (2012): »Parität und `billiges Ermessenく. Die regulierte Selbstregulierung als Mittel der Wohnungs- und Mietenpolitik im späten Kaiserreich und der jungen Weimarer Republik«, in: Collin. P. / Bender, G. /Ruppert, S. / Seckelmann, M. / Stolleis, M. (Hg.): Regulierte Selbstregulierung im frühen Interventions- und Sozialstaat, Frankfurt/M., S. 199-216.

Grimm, D. (1987): »Der Staat in der kontinentaleuropäischen Tradition«, in: ders.: Recht und Staat der bürgerlichen Gesellschaft, Frankfurt/M., S. 53-83.

Harrecker, S. (2006): Der Landwirtschaftliche Verein in Bayern 1810-1870/71, München.

Isay, R. (1920): Das Gesetz über die Regelung der Kohlenwirtschaft nebst den dazu erlassenen Ausführungs-Bestimmungen, Mannheim / Berlin / Leipzig.

Jellinghaus, L. (2006): Zwischen Daseinsvorsorge und Infrastruktur. Zum Funktionswandel von Verwaltungswissenschaften und Verwaltungsrecht in der zweiten Hälfte des 19. Jahrhunderts, Frankfurt/ M.

John, P. (1987): Handwerk im Spannungsfeld zwischen Zunftordnung und Gewerbefreiheit. Entwicklung und Politik der Selbstverwaltungsorganisationen des Handwerks bis 1933, Köln.

Käsbauer, A. (2015): Die Neuordnung der Rechtsbeziehungen zwischen Ärzten und Krankenkassen durch das Berliner Abkommen vom 23.12.1913, Baden-Baden.

Keiser, T. (2011) : »Selbstregulierung im entstehenden Nationalstaat: Autogoverno und Corpi intermedi in Italien«, in: Collin. P. / Bender, G. / Ruppert, S. / Seckelmann, M. / Stolleis, M. (Hg.): Selbstregulierung im 19. Jahrhundert - zwischen Autonomie und staatlichen Steuerungsansprüchen, Frankfurt/M., S. 127-147. 
König, K.-C. (1928): Sondergerichtsbarkeit und Schiedsgerichtsbarkeit in der bürgerlichen Rechtspflege, o. 0 .

Leifer, C. (2007): Das europäische Umweltmanagementsystem EMAS als Element gesellschaftlicher Selbstregulierung, Tübingen.

Lutterbeck, K.-G. (2011): »Französische munizipale Verwaltung als gesellschaftliche Selbstregulierung? Ansätze im Recht, in der Theorie und in der Praxis (erste Hälfte des 19. Jahrhunderts)«, in: Collin. P. / Bender, G. / Ruppert, S. / Seckelmann, M. / Stolleis, M. (Hg.): Selbstregulierung im 19. Jahrhundert - zwischen Autonomie und staatlichen Steuerungsansprüchen, Frankfurt/M., S. 87-111.

Machu, L. (2014): „Les syndicats ouvriers et le droit des conventions collectives. L'exemple de la loi du 24 juin 1936«, in : Narritsens, A. / Pigenet, M. (Hg.) : Pratiques syndicales du droit. France XXeXXIe siècles, Rennes, S. 267-277.

Mayntz, R. (2005): »Governance Theory als fortentwickelte Steuerungstheorie?«, in: Schuppert, G. F. (Hg.): Governance-Forschung. Vergewisserung über Stand und Entwicklungslinien, 2. Auflage, BadenBaden, S. 11-20.

Mayntz, R. / Scharpf, F. W. (1995): „Steuerung und Selbstorganisation in staatsnahen Sektoren«, in: dies. (Hg.): Gesellschaftliche Selbstregelung und politische Steuerung, Frankfurt/M., S. 9-38.

Moser, F. (1929): Das Rheinisch-Westfälische Kohlen-Syndikat und das Kohlenwirtschaftsgesetz, Diss. jur., Erlangen.

Nipperdey, T. (1994): Deutsche Geschichte 1800-1866. Bürgerwelt und starker Staat, München.

Nocken, U. (1981): »Korporatistische Theorien und Strukturen in der deutschen Geschichte des 19. und 20. Jahrhunderts«, in: Alemann, U. von (Hg.): Neokorporatismus, Frankfurt/M. / New York, S. 17-39.

Reese-Schäfer, W. (2007): »Nach dem Neokorporatismus«, in: ders.: Politisches Denken heute, 2. Aufl., München / Wien, S. 71-81.

Reimer, E. / Mußfeld, R. (1931): Die kaufmännischen Schiedsgerichte Deutschlands, Berlin. Reynaud, J.-D. (1997) : Les Règles du jeu : L'action collective et la régulation sociale, Paris. Rohlack, M. (2001): Kriegsgesellschaften (1914-1918), Frankfurt/M.

Rosanvallon, P. (2004) : Le modèle politique français. La société civile contre le jacobinisme de 1789 à nos jours, Paris.

Roth, R. (1997): Staat und Wirtschaft im Ersten Weltkrieg. Kriegsgesellschaften als kriegswirtschaftliche Steuerungsinstrumente, Berlin.

Roth, R. (2011): »Verflechtungen von Vereins- und Verwaltungstätigkeit als Form kommunaler Selbstregulierung «, in: Collin. P. / Bender, G. / Ruppert, S. / Seckelmann, M. / Stolleis, M. (Hg.) (2011): Selbstregulierung im 19. Jahrhundert - zwischen Autonomie und staatlichen Steuerungsansprüchen, Frankfurt/M., S. 293-309.

Roussellier, N. (1991) : L'Europe des libéraux, Paris.

Rudischhauser, S. (2014): »Regulierte Selbstregulierung im Frankreich der III. Republik: Zivilgesellschaft und »jakobinischer« Staat«, in: Collin. P. / Bender, G. / Ruppert, S. / Seckelmann, M. / Stolleis, M. (Hg.): Regulierte Selbstregulierung in der westlichen Welt des späten 19. und frühen 20. Jahrhunderts, Frankfurt/M., S. 149-188. 
Rudischhauser, S. (2016) : „Le jugement des pairs. Arbeitsrechtliche Konfliktlösungen im Frankreich der III. Republik«, in: Collin, P. (Hg.): Justice without the State within the State, Frankfurt/M.

Rudloff, W. (2012): »Politikberatung - Politikbeeinflussung - Selbstnormierung? Staatliche Beratungsgremien in Kaiserreich und Republik«, in: Collin. P. / Bender, G. / Ruppert, S. / Seckelmann, M. / Stolleis, M. (Hg.): Regulierte Selbstregulierung im frühen Interventions- und Sozialstaat, Frankfurt/M.

Ruffert, M. (2010) : § 7 »Begriff«, in: Fehling, M. / Ruffert, M. (Hg.) : Regulierungsrecht, Tübingen.

Ruppert, S. (2011): »Vereinskirche, Körperschaft oder Staatskirche - Die protestantischen Kirchen auf dem Weg zur regulierten Selbstregulierung «, in: Collin. P. / Bender, G. / Ruppert, S. / Seckelmann, M. / Stolleis, M. (Hg.): Selbstregulierung im 19. Jahrhundert - zwischen Autonomie und staatlichen Steuerungsansprüchen, Frankfurt/M., S. 311-337.

Scherner, K. O. (2011): »Handelsrecht: Selbstregulierung und Regulierung beim Eisenbahnfrachtgeschäft und im Versicherungswesen in der ersten Hälfte des 19. Jahrhunderts«, in: Collin. P. / Bender, G. / Ruppert, S. / Seckelmann, M. / Stolleis, M. (Hg.): Selbstregulierung im 19. Jahrhundert - zwischen Autonomie und staatlichen Steuerungsansprüchen, Frankfurt/M., S. 195-229.

Scheuner, U. (1978): „Staatliche Verbandsbildung und Verbandsaufsicht in Deutschland im 19. Jahrhundert «, in : »Gesellschaftliche Strukturen als Verfassungsproblem «, Der Staat. Zeitschrift für Staatslehre, öffentliches Recht und Verfassungsgeschichte (Beiheft 2), S. 97-121; frz. Übers.: »L'État, la formation et le contrôle des associations dans l'Allemagne du XIXe siècle«, in : Trivum 21/2016.

Schmidt, J. (2012): »Regulierte Selbstregulierung und Arbeiterbewegung im 19. Jahrhundert in Deutschland«, in: Collin. P. / Bender, G. / Ruppert, S. / Seckelmann, M. / Stolleis, M. (Hg.): Regulierte Selbstregulierung im frühen Interventions- und Sozialstaat, Frankfurt/M., S. 69-87.

Schmidt-Aßmann, E. (2001): »Regulierte Selbstregulierung als Element verwaltungsrechtlicher Systembildung «, in: Regulierte Selbstregulierung als Steuerungskonzept des Gewährleistungsstaates (Die Verwaltung, Beiheft 4), Berlin, S. 253-271.

Schmidt-Preuß, M. / Di Fabio, U. (1997): „Verwaltung und Verwaltungsrecht zwischen gesellschaftlicher Selbstregulierung und staatlicher Steuerung«, Veröffentlichungen der Vereinigung der Deutschen Staatsrechtslehrer, 56, Berlin / New York, S. 160-234 und S. 235-282.

Regulierte Selbstregulierung als Steuerungskonzept des Gewährleistungsstaates (2001), (Die Verwaltung, Beiheft 4), Berlin.

Schmitter, P. C. (1974): „Still the Century of Corporatism?«, The Review of Politics, 36, S. 85-131.

Schubert, W. (1999): »Das preußische Eisenbahngesetz von 1838«, Zeitschrift der Savigny Stiftung für Rechtsgeschichte, Germ. Abt., 116, S. 152-203.

Schuppert, G. F. (2015): „Selbstverwaltung und Selbstregulierung aus rechtshistorischer und governancetheoretischer Perspektive«, Max Planck Institute for European Legal History Research Paper Series No. 2015-01; http://papers.ssrn.com/sol3/papers.cfm?abstract_id=2559077, 26. März 2016.

Schuppert, G. F. (Hg.) (2005): Governance-Forschung. Vergewisserung über Stand und Entwicklungslinien, Baden-Baden.

Seckelmann, M. (2014): »Regulierte Selbstregulierung - Gewährleistungsstaat - kooperativer Staat - Governance: Aktuelle Bilder des Zusammenwirkens von öffentlichen und privaten Akteuren als Analysekategorien für historische Kooperationsformen«, in: Collin. P. / Bender, G. / 
Ruppert, S. / Seckelmann, M. / Stolleis, M. (Hg.): Regulierte Selbstregulierung in der westlichen Welt des späten 19. und frühen 20. Jahrhunderts, Frankfurt/M., S. 27-56.

Siegrist, H. (1996): Advokat, Bürger und Staat. Sozialgeschichte der Rechtsanwälte in Deutschland, Italien und der Schweiz (18.-20. Jh.), Hbd. 1, Frankfurt/M.

Simons, T. (1931): Der Aufbau der Kohlenwirtschaft nach dem Kohlenwirtschaftsgesetz vom 23. März 1919, Bonn / Köln.

Stefaniak, T. (2008): Der Wettbewerb in der Energiewirtschaft zwischen staatlicher Regulierung und selbstregulativer Verantwortung, Baden-Baden.

Stolleis, M. (2012): Geschichte des öffentlichen Rechts in Deutschland, Bd. 4, München.

Streeck, W. / Schmitter, P. C. (1999): »Gemeinschaft, Markt, Staat - und Verbände? Der mögliche Beitrag von privaten Interessenregierungen zu sozialer Ordnung «, in: Streeck, W.: Korporatismus in Deutschland. Zwischen Nationalstaat und Europäischer Union, Frankfurt/M. / New York, S. 191-222.

Terssac, G. de (Hg.) (2003): La théorie de la régulation sociale de Jean-Daniel Reynaud - Débats et prolongements, Paris.

Thoma, A. C. (2008): Regulierte Selbstregulierung im Ordnungsverwaltungsrecht, Berlin.

Trute, H.-H. (1996): „Verwaltung und Verwaltungsrecht zwischen gesellschaftlicher Selbstregulierung und staatlicher Steuerung «, Deutsches Verwaltungsblatt, S. 950-964.

Vec, M. (2006): Recht und Normierung in der Industriellen Revolution. Neue Strukturen der Normsetzung in Völkerrecht, staatlicher Gesetzgebung und gesellschaftlicher Selbstnormierung, Frankfurt/M.

Vom Feld, I. (2007): Staatsentlastung im Technikrecht. Dampfkesselgesetzgebung und -überwachung in Preußen 1831-1914, Frankfurt/M.

Voßkuhle, A. (2001): »'Regulierte Selbstregulierung` - Zur Karriere eines Schlüsselbegriffs«, in: Regulierte Selbstregulierung als Steuerungskonzept des Gewährleistungsstaates (Die Verwaltung, Beiheft 4), Berlin, S. 197-200.

\section{NOTES}

1. Vom Feld (2007), S. $204 \mathrm{ff}$.

2. Erster Teil, Kap. 3, Abschn. 1 der Vierten Verordnung des Reichspräsidenten zur Sicherung von Wirtschaft und Finanzen und zum Schutz des inneren Friedens vom 8. Dezember 1931 (RGBl. I S. 699).

3. Abkommen über die Festsetzung von Höchstzinsen für hereingenommene Gelder vom

9. Januar 1932, abgedr. in: Bank-Archiv 1931/32, S. 168.

4. Didry (1998); Machu (2014).

5. Ruffert (2010), S. 346.

6. Frison-Roche (2004), (2005).

7. Bachmann (2006); Buck-Heeb / Dieckmann (2010).

8. Allerdings findet er sich auch schon früh an prominenter Stelle in der Politikwissenschaft, so bei Streeck / Schmitter (1999), S. 208; Mayntz / Scharpf (1995).

9. Trute (1996); Schmidt-Preuß / Di Fabio (1997), Regulierte Selbstregulierung als Steuerungskonzept des Gewährleistungsstaates (2001).

10. Voßkuhle (2001).

11. Z. B. Bosch (2007); Frenzel (2007); Leifer (2007); Stefaniak (2008); Thoma (2008).

12. Reynaud (1997); de Terssac (2003). 
13. S. z. B. die Arbeiten von Alain Cottereau, Jean-Pierre Hirsch, Philippe Minard, Claire Lemercier, Alain Chatriot, Alessandro Stanziani u.a.

14. Darnaculleta i Gardella (2014), S. 71. Siehe dort auch zur Verwendung des Begriffs in anderen europäischen Ländern.

15. Siehe nur zum Überblick Schuppert (2005); Botzem u.a. (2009).

16. Braibant (2001).

17. Mayntz (2005), S. 15.

18. $\mathrm{Zu}$ Verwandtschaftsbeziehungen zwischen diesen und anderen Schlüsselbegriffen Seckelmann (2014).

19. Ähnlich auch Schuppert (2015), S. 25. f.

20. Nocken (1981).

21. Vor allem Schmitter (1974).

22. Dazu Reese-Schäfer (2007).

23. Streeck / Schmitter (1999).

24. Streeck / Schmitter (1999), S. 208, mit der Gleichsetzung von "privater Interessenregierung « und »regulierter Selbstregulierung «.

25. Scheuner (1978), S.107 f.

26. Rudischhauser (2014), S. 156.

27. Rosanvallon (2004), S. 384-395.

28. Roussellier (1991), S.48 und $94 \mathrm{f}$.

29. Siehe nur Nipperdey (1994), S. $316 \mathrm{f}$.

30. Schmidt (2012).

31. Siehe vor allem Collin u. a. (2011); Collin u. a. (2012).

32. Lutterbeck (2011); Fahrmeir (2011); Keiser (2011); Collin u. a. (2014).

33. Die Erschließung staatlich-privater Koordination unter dem Fokus »regulierte Selbstregulierung" auf anderen Gebieten, wie bei der Selbstorganisation religiöser Gemeinschaften oder bei der Kulturförderung, ist hingegen erst in Ansätzen erfolgt; siehe aber Roth (2011); Ruppert (2011).

34. S. u. Supiot, »Aktualität Durkheims«, und Lemercier, »Das heutige Frankreich«.

35. Ausführungsbestimmungen zum Gesetz über die Regelung der Kohlenwirtschaft vom 23. März 1919 vom 21. August 1919 (RGBl. S. 1449).

36. Isay (1920), S. 103; Moser (1929), S. 37 f.; Simons (1931), S. 53 f.;

37. Ausnahme waren z. B. Sicherheitsvorschriften der Berufsgenossenschaften der Unfallversicherung ( $\S 78$ ff. Unfallversicherungsgesetz vom 6. Juli 1884 (RGBl. S. 69), die nicht nur die genossenschaftsangehörigen Unternehmen banden sondern auch die betriebsangehörigen Arbeiter - dazu Ayass (2012).

38. Rudischhauser (2014), S. $181 \mathrm{ff}$.

39. Vec (2006), S. $223 \mathrm{ff}$.

40. Deparade (1927).

41. Reimer / Mußfeld (1931), S. $217 \mathrm{ff}$.

42. Scherner (2011).

43. Rudischhauser (2016).

44. Gesetz, betreffend die Krankenversicherung der Arbeiter, vom 15. Juni 1883 (RGBl. S. 73).

45. Unfallversicherungsgesetz vom 6. Juli 1884 (RGBl. S. 69).

46. Schubert (1999), S. $175 \mathrm{ff}$.

47. §§ 103e Ziffer 1, 133 Abs. 4 Gewerbeordnung i. d. F. des Gesetzes vom 26. Juli 1897 (RGBl.

S. 663).

48. Vom Feld (2007), S. $64 \mathrm{ff}$.

49. Ausführlich dazu für Deutschland Brand (1990 ff.). 
50. Für die Rechtsanwälte Siegris (1996), S. 361 ff.; für die Ärzte Gesetz, betreffend die ärztlichen Ehrengerichte, das Umlagerecht und die Kassen der Ärztekammern, vom 25. November 1899 (PrGS S. 565).

51. Im Unterschied zum »klassischen« Schiedsgericht, das im 10. Buch der Civilprozeßordnung vom 30. Januar 1877 (RGBl. S. 83) geregelt war und dessen Regeln vom Modell eines durch ad hoc Parteivereinbarung gegründeten Gerichtes ausgingen, handelte es sich im hier behandelten Zusammenhang um ständige Gerichte, die aufgrund von Verbandsvereinbarungen oder staatlichen Gesetzen gebildet worden waren.

52. Z.B. Einigungsstellen für Vertragsstreitigkeiten zwischen Ärzteverbänden und Krankenkassen auf der Grundlage des Abkommen zwischen den Organisationen der Ärzte und der Krankenkassen, betreffend das Verhältnis zwischen Ärzten und Krankenkassen, vom 23. Dezember 1913 (Beilage Nr. 5 des Ministerialblatts der Handels- und Gewerbeverwaltung 1914, S. 85 ff.); Schiedsgerichte für Streitigkeiten zwischen Sparkassen, Kreditgenossenschaften und Banken auf der Grundlage des Abkommens auf dem Gebiet des Wettbewerbs vom Januar 1932 (abgedruckt in: Jahrbuch des Deutschen Sparkassen- und Giroverbandes 1931/32, S. 175 ff.)

53. Ayass (2016).

54. Z. B. Mieteinigungsämter, Führer (2012); Einigungsstellen über Heizenergieversorgung, Verordnung über Sammelheizungs- und Warmwasserversorgungsanlagen in Mieträumen vom 2.

November 1917 (RGBl. S. 989); Verordnung über Sammelheizungs- und Warmwasserversorgungsanlagen vom 22. Juni 1919 (RGBl. S. 595); Pachteinigungsämter, König (1928), S. 35 ff.

55. Schmidt-Aßmann (2001), S. 261; Di Fabio (1997), S. 269.

56. Sieht man davon ab, dass ein Verein für seine Rechtsfähigkeit der staatlichen Genehmigung bedurfte.

57. So vor allem Landwirtschaftsvereine, Harrecker (2006), oder Gesundheitsvereine, Jellinghaus (2006), S. $98 \mathrm{ff}$.

58. Streeck / Schmitter (1999), S. 204.

59. Vom Feld (2007).

60. Dies betraf die Kali- und die Kohlenwirtschaft sowie die metallurgische Industrie.

61. John (1987).

62. Käsbauer (2015).

63. Rudloff (2012); Chatriot / Lemercier (2008).

64. Roth (1997); Rohlack (2001).

65. Stolleis (2012), S. $367 \mathrm{ff}$.

66. Grimm (1987), S. 69 f.

INDEX

Schlüsselwörter : regulierte Selbstregulierung, Einleitung, Staat, Gesellschaft, Neokorporatismus, Governance

Mots-clés : autorégulation régulée, introduction, État, société, néo-corporatisme, gouvernance 


\section{AUTEURS}

\section{PETER COLLIN}

Peter Collin ist wissenschaftlicher Mitarbeiter am Max-Planck-Institut für europäische Rechtsgeschichte (Frankfurt/M.). Nähere Informationen finden Sie hier.

\section{SABINE RUDISCHHAUSER}

Sabine Rudischhauser ist wissenschaftliche Mitarbeiterin am Centre de recherche Mondes modernes et contemporains der Freien Universität Brüssel. Nähere Informationen finden Sie hier. 